\title{
Straatsma Syndrome - Report of Two Cases
}

\author{
Pedro Manuel Baptista ${ }^{1,2 *}$, João Heitor Marques ${ }^{1}$, Natália \\ Ferreira ${ }^{1}$, Luísa Malheiro ${ }^{1}$, Sofia Maia ${ }^{1}$, Vasco Miranda ${ }^{1,2}$, Ricardo \\ Parreira $^{1}$ and Pedro Menéres ${ }^{1,2}$ \\ ${ }^{1}$ Ophthalmology Department, Centro Hospitalar Universitário do Porto, Porto, \\ Portugal \\ ${ }^{2}$ Instituto de Ciências Biomédicas Abel Salazar, Porto, Portugal \\ *Corresponding Author: Pedro Manuel Baptista, Ophthalmology Department, \\ Centro Hospitalar Universitário do Porto, Porto, Portugal.
}

Received: February 05, 2021

Published: February 16, 2021

(C) All rights are reserved by Pedro Manuel Baptista., et al.

\begin{abstract}
Introduction: Straatsma syndrome is a rare disease characterized clinically by the exuberant persistence of myelin fibers in the retina associated or not with the presence of myopia, strabismus and amblyopia. The present study is aimed to describe two cases of this entity.

Methods: Retrospective patient's clinical records review.

Results: In case 1, a 17-year-old male was referred to the ophthalmology consultation due to decreased visual acuity in the right eye (RE). With no relevant systemic or ophthalmological history, he had a better corrected visual acuities (BCVA) of 20/200 in the RE and 20/20 without correction in the left eye (LE), with no oculomotricity changes and fundoscopy revealed the persistence of myelinated retinal nerve fibers (MRNF) near the upper temporal arch and myopic choroid in the RE and no alteration in the LE. In case 2, a 39-year-old male with family history of myopia was referred with a diagnosis of rhegmatogenous retinal detachment on the LE. On ophthalmic examination, BCVA were 20/20 on the RE and hand motion on the LE. During the pars plana vitrectomy, a total rhegmatogenous retinal detachment was observed in the LE, with MRNF accompanying the temporal arcades and a macular hole $(\mathrm{MH})$. After three pars plana vitrectomies, including an inverted ILM flap technique attempted to close the MH, and eight months after silicone oil extraction, the aphakic LE presented a 20/200 BCVA with the retina fully attached and the MH closed.

Conclusion: Despite some controversy in its features, Straatsma syndrome should be included in the differential diagnosis of leukocoria and must be suspected in the presence of refractive errors with anisometropia. The general prognosis is poor to moderate, but better results can be achieved with a prompt diagnosis and amblyopia management.
\end{abstract}

Keywords: Myelinated Retinal Nerve Fibers (MRNF); Straatsma Syndrome; Macular Hole (MH); Anisometropia; Amblyopia; Strabismus; Better Corrected Visual Acuities (BCVA)

\section{Introduction}

Myelinated retinal nerve fibers (MRNF) are rare anomalies that appear as grey-white opaque lesions on the retina with feathery edges that obscure retinal details. They may be located on the disc, more often at the temporal inferior, followed by the temporal su-

Citation: Pedro Manuel Baptista., et al. "Straatsma Syndrome - Report of Two Cases". Acta Scientific Ophthalmology 4.3 (2021): 56-61. 
perior sector of the nerve head [1]. Besides congenital in the vast majority of cases, there are few reports of acquired cases [2].

The association between unilateral MRNF, ipsilateral myopia and amblyopia was described many years ago by Straastma., et al [3]. The present study is aimed to describe two cases of this entity.

\section{Methods}

Retrospective patient's clinical records review. The study followed the tenets of the Declaration of Helsinki. Informed consent for the publication of anonymized clinical data including clinical images was signed by the patients.

\section{Results}

Case 1

Male, 17 years old, referred to an ophthalmology appointment due to chronic diminished best corrected visual acuity (BCVA, Snellen notation) on the right eye (RE). On the evaluation, the patient denied history of personal or familiar systemic medical problems. Personal, but not familiar history of ametropia corrected with glasses four years before was referred.

On the ophthalmic examination, BCVA's were 20/200 on the RE, with spherocylindrical correction of $-13.00-5.00 \times 90^{\circ}$ and 20/20 on the left eye (LE) without correction. There were no alterations found both in the oculomotricity exam and biomicroscopy. Relative afferent pupillary defect (RAPD) was absent.

On the fundoscopy, MRNF were found within the peripapillary area and following the path of the superior temporal vascular arcade (Figure 1). No alterations were found in the LE (Figure 1).

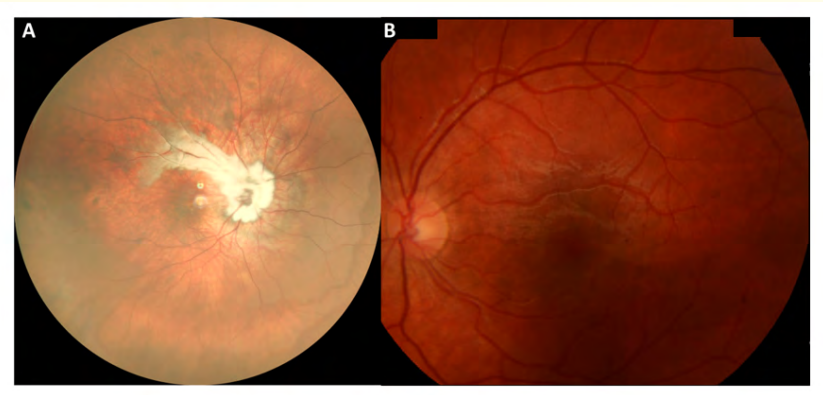

Figure 1: A: Wide-field fundus photography, right eye; B: Fundus photography, left eye.
Spectral domain optical coherence tomography (SD-OCT, Spectralis, Heidelberg ${ }^{\circledR}$ ) exam revealed macular signs of pathologic myopia (Figure 2) and increased peripapillary retinal nerve fiber layer thickness (ppRNFLT) but with improper segmentation in the RE. No macular (Figure 2) or optic disk alterations were found in the LE.

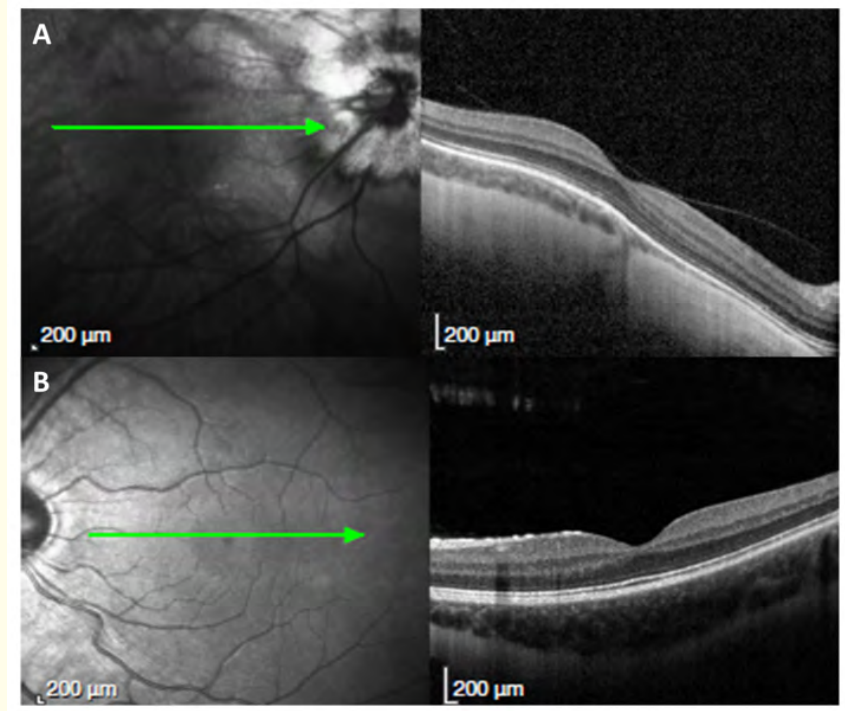

Figure 2: Foveal-centered SD-OCT scan from the right eye (A) and left eye (B).

The visual field (VF) exam (Humphrey Visual Field Analyser $3^{\circledR}$, III white stimulus, 31.5 asb background, 10-2 SITA-fast strategy) of the RE showed general loss of retinal sensitivity (MD -8.48dB, PSD 2.23dB). The LE exam showed no alterations. In the Esterman Binocular Suprathreshold Test (EBST) (Humphrey Visual Field Analyzer $3^{\circledR}$; central fixation target, white III stimulus, 31.5asb background, two zone strategy, age-corrected, central and peripheral references of $34 \mathrm{~dB}$ ) the total non-viewed points were $62 / 120$ (Figure 3).

Case 2

Male, 39 years old, referred from another public hospital with a diagnosis of rhegmatogeneous retinal detachment on the LE. On the evaluation, the patient denied history of personal or familiar systemic medical problems. Personal, but not familiar history of 


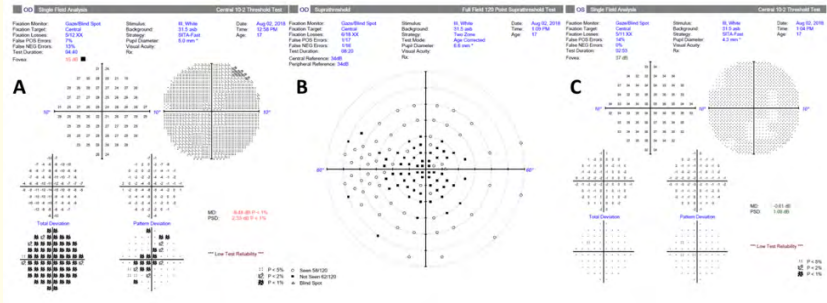

Figure 3: A: Monocular visual field test, right eye; B: Esterman binocular visual field test; C: Monocular visual field test, left eye.

ametropia corrected with glasses since childhood and occlusion treatment in the other eye were referred.

On the ophthalmic examination, BCVA's were 20/20 on the RE, with spherocylindrical correction of -1.00 and hand motion on the LE. There were no alterations found both in the oculomotricity exam and biomicroscopy. Relative afferent pupillary defect (RAPD) was absent. Axial length was $23.91 \mathrm{~mm}$ in the RE and $30.84 \mathrm{~mm}$ in the LE. Objective refraction was $-1.00-0.50 \times 10^{\circ}$ in the RE and impossible to address in the LE.

On the fundoscopy, no alterations were found in the RE and a total rhegmatogenous retinal detachment (RRD) was observed in the LE.

The patient was submitted to pars plana vitrectomy (PPV) with posterior lensectomy. During the surgery, peripapillary MRNF and a macular hole $(\mathrm{MH})$ were observed, but inner limiting membrane (ILM) peel was not possible, due to decreased visualization in the zone of the MRNF and anatomic conditions of the eye. Laser photocoagulation ( $360^{\circ}$ ), including zones of peripheral degeneration and the rhegmatogenous lesion, was performed and the tamponade was made with perfluoropropane (C3F8). Two weeks after the procedure, a partial re-detachment was diagnosed and the MRNF within the peripapillary area and following the path of both temporal vascular arcades were confirmed (Figure 4). The SD-OCT showed typical signs of myopic maculopathy with posterior staphyloma and confirmed the MH (Figure 5). A new PPV was performed, through a temporal approach in order to improve the access to the macula, and an inverted ILM flap technique attempted to close the $\mathrm{MH}$, followed by silicon oil 1000 tamponade. In the early postoperative period, a rise in the intraocular pressure (IOP) was observed and the silicon oil was then removed through a new PPV with additional ciliary processes photocoagulation. Eight months after silicone oil extraction, the aphakic LE presented a 20/200 BCVA with spherocylindrical correction of $+1.00-1.50 \times 170^{\circ}$ and stable IOP of $19 \mathrm{mmHg}$ without medication. The retina was fully attached, the MH was closed (Figure 6) and the MRNF were visible as described (Figure 7).

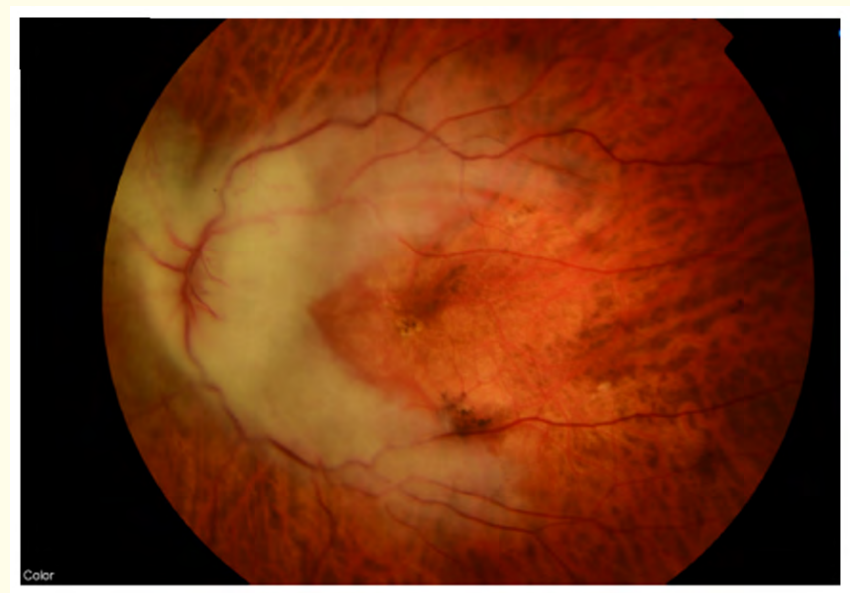

Figure 4: Fundus photography, left eye.

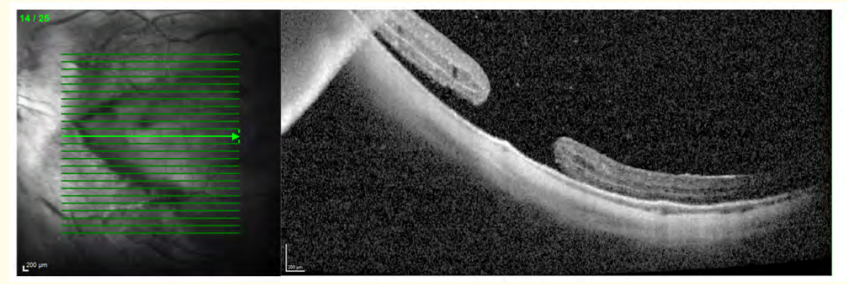

Figure 5: Foveal-centered SD-OCT scan from the left eye.

\section{Discussion}

Although the pathogenesis of MRNF is not known, it is believed that can result from an imbalance between the formation of the lamina cribosa, which proceeds posteriorly from the limbus and 


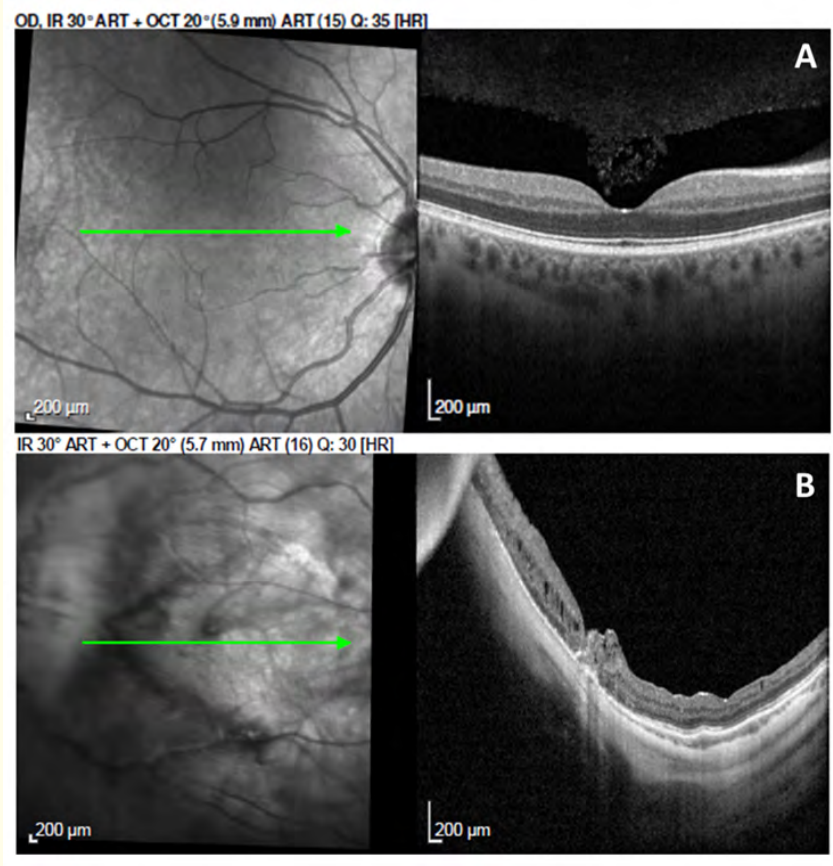

Figure 6: Foveal-centered SD-OCT scan from the right eye (A) and left eye (B).

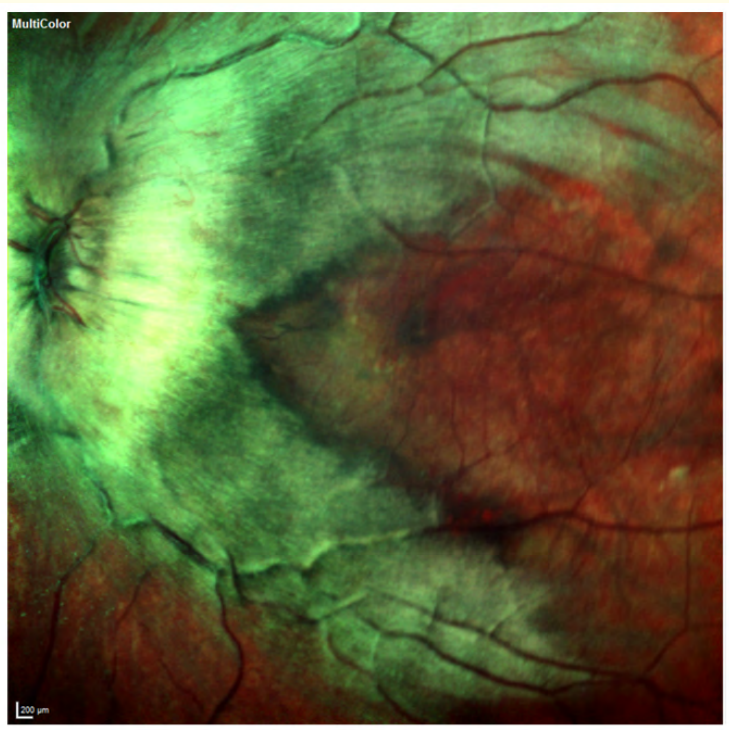

Figure 7: Multicolor fundus photography, left eye. the process of myelination, which begins from the lateral geniculate body [4]. Oligodendrocytes are effectors in the myelination of the ganglion cell axons and impaired astrocytes function of impeding those cells to migrate through lamina cribosa can is taught to play a role [5]. As this process occurs early in the eye development, it explains.

In a study of 3968 autopsies, Straatsma., et al. [6] found MRNF in 39 cases. From these, 4 had associated history of ipsilateral myopia, amblyopia, strabismus. Myopia has been reported in 35\% to $58 \%$ in patients with myelination of fibers in previous studies, $83 \%$ with refraction of up to 6 diopters [7]. One hypothesis for this association is that a blurred image on the retina produces visual depravity at a critical stage of ocular development, which could be the cause of axial elongation of the eye and development of myopia, which in turn delays the development of the lamina cribrosa, thus following an extension of myelination of fibers through the retina [4].

More recently, the aforementioned associations were called into question as an entity called Reverse Straatsma Syndrome was described in hyperopic patients, including amblyopia with bilateral myelination of the retinal nerve fibers, particularly extensive in the eye with greater hypermetropia and without exotropia [8].

In the present study, case 1 had unilateral myopia with high grade anisometropia and the case 2 , despite lack of preoperative objective refraction information, had $6 \mathrm{~mm}$ of difference in the axial length between both eyes, with the longer one compatible with axial high myopia and the other near the inferior limit.

While we can't make BCVA comparisons with the case 2 due to the lack of information before the RRD, case 1 showed BCVA in line with the reported $[4,8,9]$. It is of notice that a refractive difference of $1.25 \mathrm{D}$ or greater in hyperopes is generally required for the development of amblyopia, unlike myopes, where an even greater difference seems to be required and this is highlighted in the case of Reverse Straatsma [8].

Despite difficult optic nerve analysis in most eyes with peripapillary MRNF, associated optic nerve affection, namely in the form of optic nerve hypoplasia, can be rarely associated [9]. In our cases, good optic nerve function was proven by the absence of RAPD. 
The type and amount of the refractive error and anisometropia seems to have a stronger influence on the relative visual acuity of each patient's eyes and subsequent amblyopia than the presence of retinal nerve fiber myelination $[8,9]$. In fact, scotomas in myelinated retinas are smaller than predicted by the extent of myelination, suggesting that light penetrates to the photoreceptor layer despite the myelin interference $[6,9,10]$. In the presented case 1 , both the monocular 10.2 and the binocular VF revealed decreased general sensitivities, without clearly defined scotomas with possible correlation with the MRNF, which is in line with the aforementioned.

The inclusion of some form of oculomotricity impairment, namely exotropia, described initially by Straatsma is controversial and a more recent review found it not ubiquitous, correlating it with the amount of myopic anisometropia and visual acuity. Besides high differences between eyes and poor visual acuity in the affected one, ocular motility impairment was not apparent in our cases.

Recently, the prevalence of MRFN was reported to be $0.4 \%$ within the 4439 patients enrolled in the Beijing Eye Study [11], with lack of association with factors like age, gender, refractive error, visual acuity (VA), intraocular pressure, cataract, glaucoma or agerelated macular degeneration. However, associations with other ocular conditions like Keratoconus or Coloboma [9] and systemic diseases like neurofibromatosis, Down syndrome and Craniofacial Dysostosis were reported [6]. Additionally, familial inheritance was also described [12]. In the present study, none of the cases had any other associations.

The prognosis of this entity is poor to moderate, particularly if the condition is not diagnosed during the amblyogenic period, giving rise to a picture of severe amblyopia. Due to its rarity and features not widely known, Straatsma syndrome very often goes undiagnosed. Therefore, the inclusion in the differential diagnosis of leukocoria is very important [13]. The presented cases are two examples of a late diagnosis, with very poor prognosis. Additionally, given the association to high degrees of myopia in the majority of cases, the risk of RRD and other high myopia associated problems like $\mathrm{MH}$ have to be monitored and with a high level of suspicion, due to the possible mask effect of amblyopia, as highlighted in the presented case 2 .

When the diagnosis is made early in life, and after the exclusion of associated alterations, patients should undergo ametropia cor- rection and aggressive amblyopia therapy, because unexpectedly good visual results can be achieved as described by Kee and Hwang [14], with initiation of therapy at a mean age of 4.2 years. The authors found a final BCVA of 20/30 in 4 out of 12 children and highlighted the initial refractive error, the amount of anisometropia, the area of myelination and the appearance of the macula as the main prognostic factors.

Measures to detect and allow adequate treatment in order to maximize the visual potential of patients like these are needed. Therefore, governmental programs aimed to decrease the prevalence of amblyopia are nuclear in order to have a competitive society in a global world. In Europe there is the EUSCREEN (https:// www.euscreen.org/), including projects in several countries, of which the pilot program launched in 2016 by the Portuguese government in the north of Portugal - Rastreio de Saúde Visual Infantil da ARS Norte (http://portaisars.azurewebsites.net/norte/wp-content/uploads/sites/3/2017/12/Programa_RSVI_Relatorio_Final. pdf) is an example of success.

\section{Conclusion}

Straatsma syndrome is a rare entity. Despite some controversy in its diagnostic features, it should be included in the differential diagnosis of leukocoria and must be suspected in the presence of refractive errors with anisometropia. The general prognosis is poor to moderate, but better results can be achieved with a prompt diagnosis and amblyopia management.

\section{Bibliography}

1. Kodama T., et al. "Myelinated retinal nerve fibers: prevalence, location and effect on visual acuity". Ophthalmologica Journal International D'ophtalmologie International Journal of Ophthalmology Zeitschrift fur Augenheilkunde 200 (1990): 77-83.

2. Prakalapakorn SG and Buckley EG. "Acquired bilateral myelinated retinal nerve fibers after unilateral optic nerve sheath fenestration in a child with idiopathic intracranial hypertension". Journal of AAPOS: the Official Publication of the American Association for Pediatric Ophthalmology and Strabismus 16 (2012): 534-538.

3. Straatsma BR., et al. "Myelinated retinal nerve fibers associated with ipsilateral myopia, amblyopia, and strabismus". American journal of ophthalmology 88 (1979): 506-510. 
4. Lee MS and Gonzalez C. "Unilateral peripapillary myelinated retinal nerve fibers associated with strabismus, amblyopia, and myopia”. American Journal of Ophthalmology 125 (1998): 554-556.

5. Ffrench-Constant C., et al. "Evidence that migratory oligodendrocyte-type- 2 astrocyte (0-2A) progenitor cells are kept out of the rat retina by a barrier at the eye-end of the optic nerve". Journal of Neurocytology 17 (1988): 13-25.

6. Straatsma BR., et al. "Myelinated retinal nerve fibers". American Journal of Ophthalmology 91 (1981): 25-38.

7. Ellis GS., et al. "Myelinated nerve fibers, axial myopia, and refractory amblyopia: an organic disease". Journal of Pediatric Ophthalmology and Strabismus 24 (1987): 111-119.

8. Shenoy R., et al. "Bilateral Hypermetropia, Myelinated Retinal Nerve Fibers, and Amblyopia". Middle East African Journal of Ophthalmology 18 (2011): 65-66.

9. Tarabishy AB., et al. "Syndrome of myelinated retinal nerve fibers, myopia, and amblyopia: a review". Survey of Ophthalmology 52 (2007): 588-596.

10. Munteanu M., et al. "[Unilateral myelinated nerve fibers associated with myopia or amblyopia]". Oftalmologia 53 (2002): 61-66.

11. You Q., et al. "Prevalence of myelinated retinal nerve fibres in urban and rural adult Chinese populations: the Beijing Eye Study". Acta Ophthalmologica Scandinavica 85 (2007): 631632.

12. Funnell CL., et al. "Familial myelinated retinal nerve fibres". Eye 17 (2003): 96-97.

13. Nogueira G., et al. "Straatsma syndrome: two case reports \%]". Revista Brasileira de Oftalmologia 76 (2017): 262-264.

14. Kee C and Hwang J-M. "Visual prognosis of amblyopia associated with myelinated retinal nerve fibers". American Journal of Ophthalmology 139 (2005): 259-265.

\section{Assets from publication with us}

- Prompt Acknowledgement after receiving the article

- Thorough Double blinded peer review

- Rapid Publication

- Issue of Publication Certificate

- High visibility of your Published work

Website: www.actascientific.com/

Submit Article: www.actascientific.com/submission.php

Email us: editor@actascientific.com

Contact us: +919182824667 